\title{
Impacto da facectomia na qualidade de vida de idosos atendidos em campanha
}

\section{assistencial de catarata}

Tainara Sardeiro de Santana ${ }^{1}$, Marcos Pereira de Ávila ${ }^{2}$, David Leonardo Cruvinel Isaac ${ }^{3}$, Gabriela Camargo Tobias ${ }^{4}$, Thatianny Tanferri de Brito Paranaguá ${ }^{5}$

\footnotetext{
${ }^{1}$ Enfermeira, Mestre em Ciências da Saúde. Discente do Programa de PósGraduação em Ciências da Saúde da Universidade Federal de Goiás, nível Doutorado. Enfermeira da Fundação de Apoio ao Hospital das Clínicas da Universidade Federal de Goiás. Goiânia, GO, Brasil. E-mail: enftainara@gmail.com.

${ }^{2}$ Médico, Doutor em Oftalmologia. Professor Titular da Universidade Federal de Goiás. Goiânia, GO, Brasil. E-mail: marcosavila@cbco.com.br.

${ }^{3}$ Médico, Doutor em Ciências da Saúde. Professor Adjunto da Universidade Federal de Goiás. Goiânia, GO, Brasil. E-mail: cruvinelisaac@hotmail.com.

${ }^{4}$ Enfermeira, Mestre em Enfermemagem. Discente do Programa de Pós-Graduação em Medicina Tropical da Universidade Federal de Goiás, nível Doutorado. Enfermeira do Secretaria Municipal de Saude de Senador Canedo. Goiânia, GO, Brasil. E-mail: gabicamargo22@gmail.com.

${ }^{5}$ Enfermeira, Doutora em Enfermagem. Professora Adjunto da Faculdade de Ciências da Saúde da Universidade de Brasília. Brasília, DF, Brasil. E-mail:

ttb.paranagua@gmail.com.
}

Recebido: 22/01/2016.

Aceito: 01/06/2017.

Publicado: 22/08/2017.

\section{Como citar esse artigo:}

Santana TS, Ávila MP, Isaac DLC, Tobias GC, Paranaguá TTB. Impacto da facectomia na qualidade de vida de idosos atendidos em campanha assistencial de catarata. Rev. Eletr. Enf. [Internet]. 2017 [acesso em: ____;19:a35. Disponível em: http://dx.doi.org/10.5216/ree.v19.39498.

\section{RESUMO}

Objetivou-se avaliar a melhora da acuidade visual (AV) antes e no $15^{\circ}$ dia do pós-operatório de facectomia e o seu impacto na qualidade de vida dos idosos. Estudo prospectivo, desenvolvido com 156 pacientes submetidos à facectomia em campanha oftalmológica. De acordo com a tabela de Snellen, a AV média, em logMAR, após 15 dias da cirurgia melhorou de 1,23 para 0,57 nos idosos $(p=0,000)$. Na comparação das médias de qualidade de vida, pelo questionário de avaliação da qualidade de vida Visual Functioning Questionnaire (VQF-25), o subdomínio visão geral apresentou a maior diferença entre as médias antes e após a facectomia (de 29,65 para 89,87). A análise de correlação apontou que a melhora da AV melhorou a satisfação geral, em relação à atividade para longe, dor ocular, saúde mental e dependência, levando a um impacto positivo na qualidade de vida dos idosos. Levantaram-se indicadores que direcionam à terapêutica mais individualizada.

Descritores: Catarata; Qualidade de Vida; Acuidade Visual; Facoemulsificação; Promoção da Saúde.

\section{INTRODUÇÃO}

A catarata é definida como qualquer opacidade do cristalino que difrate a luz, acarretando efeito negativo na visão ${ }^{(1)}$, sendo a principal causa de cegueira no Brasil( ${ }^{(2)}$. De acordo com o Conselho Brasileiro de Oftalmologia, calcula-se que $10 \%$ da população acima de 50 anos de idade tenha catarata, e essa prevalência se eleva para $50 \%$ no grupo 
etário de 65 a 74 anos de idade e para $75 \%$ após $75 \operatorname{anos}^{(3)}$.

Estudo realizado nos Estados Unidos verificou que as condições crônicas sofridas pelos idosos, em ordem de prevalência, incluem a hipertensão $(36,1 \%)$, a doença pulmonar obstrutiva crônica $(23,7 \%)$ e a catarata $(16,7 \%)^{(4)}$. Em Honduras, a catarata $(59,2 \%)$ e o glaucoma $(21,1 \%)$ são as principais causas de cegueira entre os idosos ${ }^{(5)}$. No nordeste brasileiro a catarata é a doença ocular mais prevalente (45\%) entre os idosos, seguida pelo glaucoma $(18 \%)^{(6)}$.

Embora haja avanços na cirurgia e no acesso aos serviços de saúde, em muitas partes do mundo, a catarata permanece como a maior causa de cegueira evitável cirurgicamente ${ }^{(2)}$. Dentre os procedimentos eletivos, a facectomia consiste na remoção da catarata, geralmente associada ao implante de uma lente intraocular, em substituição a lente ocular natural que se tornou opaca (cristalino). Essa cirurgia apresenta alta eficiência e custo-benefício favorável, tanto no tratamento quanto na reabilitação visual ${ }^{(7)}$, intervindo na diminuição da acuidade visual, influenciando a qualidade de vida das pessoas ${ }^{(2,8)}$ e reduzindo prejuízos funcionais para os pacientes ${ }^{(9)}$.

A saúde e a qualidade de vida dos idosos, mais que em outros grupos etários, sofrem influência de múltiplos fatores, tais como físicos, psicológicos, sociais e culturais. Assim, a assistência ao idoso deve considerar os processos de perdas próprias do envelhecimento e as possibilidades de prevenção, manutenção e reabilitação do seu estado de saúde ${ }^{(10)}$. A saúde ocular interfere em todos esses fatores. Dentre os principais aspectos a serem melhorados pela facectomia podem ser citados a independência, a autonomia, a possibilidade de lazer e leitura, além de, em muitos casos, restaurar a alegria de viver e a motivação para novas experiências de vida. Frente a isso, há a necessidade de estimar qual o real impacto dessa intervenção na qualidade de vida dos idosos.

Reconhece-se o efeito da facectomia na melhora da acuidade visual, porém, numa busca minuciosa nas bases de dados disponíveis, não foram encontrados trabalhos na literatura científica brasileira que abordem a influência dessa intervenção na qualidade de vida dos idosos com catarata, especialmente utilizando instrumento validado sobre qualidade de vida. Desse modo, diante da tendência de crescimento dessa população no Brasil e no mundo, este estudo tem por objetivo comparar a acuidade visual antes e no $15^{\circ}$ dia pós-operatório da facetomia e descrever o impacto desta intervenção na qualidade de vida dos idosos.

\section{MÉTODO}

Estudo prospectivo, com abordagem quantitativa, descritiva-correlacional, desenvolvido em um hospital público localizado em Macapá-AP.

A população foi composta por 156 idosos, com indicação para a realização de cirurgia de catarata, inscritos no Projeto Visão para Todos em uma campanha assistencial realizada por um convênio entre a Secretaria Estadual de Saúde do Amapá e o Centro de Referência em Oftalmologia do Hospital das Clínicas da Universidade Federal de Goiás (CEROF/HC/UFG). A região do Amapá não possui equipamentos e 
profissionais especializados para o procedimento de facectomia pelo Sistema Único de Saúde (SUS), o que justifica o convênio do Estado com o CEROF/HC/UFG, no intuito de suprir necessidades de saúde locais. A seleção dessa população para o estudo foi motivada pela participação de uma das pesquisadoras no Projeto Visão para Todos, participando da campanha no contexto local de Macapá e estar vinculada ao CEROF/HC/UFG.

Foram incluídos no estudo idosos com idade maior ou igual a 60 anos e excluídos aqueles que apresentavam algum comprometimento na função mental e cognitiva que impossibilitasse o preenchimento do questionário.

Os dados foram coletados entre setembro e outubro de 2013 , utilizando a tabela de Snellen para verificação da acuidade visual, e o questionário de avaliação da qualidade de vida, Visual Functioning Questionnaire (VQF-25) desenvolvido pelo National Eye Institute (NEI) ${ }^{(11)}$, traduzido para língua portuguesa ${ }^{(12)}$ com validade e confiabilidade estatisticamente comprovadas ${ }^{(13)}$, antes da facectomia e no 15 o dia pósoperatório.

O VFQ-25 avalia a saúde geral do paciente e a qualidade de vida relacionadas à acuidade visual. Ele possui 25 questões agrupadas em 12 subdomínios com uma ou mais questões. Para cada questão há cinco possibilidades de resposta, sendo que para cada resposta obtém-se uma pontuação que varia de zero a 100. A pontuação final obtida é então dividida pelo número de questões, obtendo-se um escore para cada paciente cujo valor mínimo é zero e o valor máximo é 100. Quanto maior o escore alcançado, melhor é a percepção da qualidade de vida e função visual do paciente.

Os dados foram analisados utilizando o software Statistical Package for the Social Sciences (SPSS versão 18.0) e então foi realizada análise estatística descritiva dos dados sociodemográficos. Utilizou-se o teste de Wilcoxon para a comparação da acuidade visual e dos subdomínios do questionário de VFQ-25 antes e após a cirurgia. Na comparação das características dos participantes e na satisfação geral com a saúde, utilizou-se o teste $t$ Student pareado. Para a correlação da acuidade visual com os domínios do questionário VFQ-25 após a cirurgia, utilizou-se o coeficiente de correlação de Spearman, pois se verificou distribuição não paramétrica dos dados. Para a interpretação do coeficiente de correlação, seja positivo ou negativo, os seguintes valores de referências foram adotados: 0,00 a 0,19 representa correlação muito fraca; 0,20 a 0,39, correlação fraca; 0,4 a 0,69, correlação moderada; 0,70 a 0,89, correlação forte; e 0,9 a 1,00, correlação muito forte ${ }^{(14)}$. $O$ valor de $p<0,05$ foi considerado estatisticamente significativo em todos os testes.

O estudo foi aprovado pelo Comitê de Ética em Pesquisa Médica, Humana e Animal do Hospital das Clínicas da Universidade Federal de Goiás CEPMHA/HC/UFG parecer n 569.338. Todos os participantes consentiram com a pesquisa e foi garantido o anonimato. Demais aspectos éticos seguiram a Resolução CNS №466/12.

\section{RESULTADOS}

Participaram do estudo 156 idosos submetidos à cirurgia de facectomia. Dentre os participantes, 80 
(51,3\%) eram do sexo feminino e $76(48,7 \%)$, do sexo masculino. A idade variou de 60 a 95 anos, com 84 $(53,8 \%)$ idosos na faixa etária de 70 anos ou mais e $72(46,2 \%)$ idosos com idade entre 60 a 69 anos, e a média de idade foi de 66,6 anos.

Quanto ao estado civil, 130 (80,3\%) pacientes estavam sem companheiro (solteiro, viúvo, divorciado) e $26(16,7 \%)$ com companheiro (casado/amasiado). Destaca-se que $47(30,1 \%)$ eram analfabetos e 109 $(69,9 \%)$ tinham alguma escolaridade e, dentre esses, 57 (36,5\%) tinham apenas o fundamental incompleto.

A situação previdenciária para 141 (90,4\%) pacientes era de aposentados e apenas 15 (9,6\%) trabalhavam. A renda mensal variou de nenhum a 4,7 salários mínimos, sendo que $148(94,9 \%)$ relataram receber até um salário mínimo, que equivalia a $\mathrm{R} \$ 678,00$ no período do estudo.

A acuidade visual média, em logMAR, após 15 dias da cirurgia, passou de 1,23 $( \pm 0,46)$ para 0,57 $( \pm 0,53)$, obtendo diferença estatisticamente significativa $(p=0,000)$, o que confirma a melhora da acuidade visual após a intervenção cirúrgica.

A Tabela 1 mostra a comparação da qualidade de vida dos idosos antes e após a facectomia.

Tabela 1: Comparação dos escores de qualidade de vida dos idosos antes e após a facectomia. Macapá, AP, Brasil, 2013.

\begin{tabular}{cccccc}
\hline \multirow{2}{*}{ Subdomínios } & \multicolumn{2}{c}{ Antes (N=156) } & \multicolumn{2}{c}{ Depois (N=156) } & \multirow{2}{*}{ Wilcoxon p-valor } \\
\cline { 2 - 5 } & Média & Desvio padrão & Média & Desvio padrão & \\
\hline Saúde geral & 29,65 & 27,50 & 37,18 & 27,75 & 0,009 \\
Visão & 29,97 & 25,31 & 89,87 & 17,52 & 0,018 \\
Dor ocular & 65,30 & 26,01 & 72,92 & 23,06 & 0,000 \\
Atividade perto & 64,57 & 20,98 & 70,73 & 20,54 & 0,001 \\
Atividade longe & 69,62 & 20,98 & 76,75 & 22,01 & 0,000 \\
Aspecto social & 90,00 & 16,69 & 95,00 & 12,62 & 0,001 \\
Saúde mental & 56,41 & 21,92 & 68,23 & 18,90 & 0,000 \\
Atividades diárias & 54,73 & 29,08 & 60,90 & 27,45 & 0,008 \\
Dependência & 62,39 & 23,02 & 74,20 & 18,47 & 0,000 \\
Capacidade de dirigir & 5,77 & 18,67 & 6,03 & 20,75 & 0,547 \\
Visão de cores & 82,82 & 22,66 & 89,87 & 17,52 & 0,000 \\
Visão periférica & 77,05 & 23,26 & 82,69 & 21,20 & 0,003 \\
Satisfação geral & 57,35 & 14,49 & 64,25 & 12,46 & 0,000 \\
\hline
\end{tabular}

Na comparação das médias de qualidade de vida antes e após a facectomia, com exceção do subdomínio capacidade para dirigir automóveis, observou-se diferença estatisticamente significante em todos os subdomínios do questionário. Em todos esses casos, verifica-se que a qualidade de vida foi melhor após a realização da cirurgia.

A maior alteração de média de satisfação obtida pelos pacientes foi em relação à visão geral, que antes da cirurgia apresentou escore de satisfação de 29,65 e, após a cirurgia, aumentou para 89,87. Outras médias altas observadas foram nos domínios "dependência" e "saúde geral".

As dimensões saúde mental e atividades diárias, apesar de significativas, apresentaram escores de 68,23 e 60,9, respectivamente, indicando área que necessita de maior apoio da equipe de saúde para melhorar o grau de satisfação do idoso frente às ações vinculadas às respectivas dimensões.

Em relação às atividades diárias, cabe ressaltar que no período de investigação os pacientes ainda se 
encontravam com algumas restrições de força, próprias do pós-operatório em oftalmologia.

A capacidade de dirigir, com escore de 6,03 após a facectomia, é uma dimensão que apresenta divergência de todas as outras, e que se vincula a aspectos sociais, econômicos, culturais, dentre outros, sugerindo que essa percepção pode não estar relacionada apenas à qualidade da visão. De todo modo, a satisfação geral com a visão, tanto antes como depois da facectomia, com escores de 57,35 e 64,25, respectivamente, recebeu interferência do domínio capacidade de dirigir.

Ressalta-se, ainda, a percepção de saúde geral do idoso, que apesar de apresentar melhora significativa após a facectomia, apresenta baixos escores $(29,65$ e 37,18, antes e depois da cirurgia, respectivamente).

A Tabela 2 apresenta a comparação entre as características dos pacientes e a satisfação geral com o funcionamento visual após a cirurgia.

Tabela 2: Comparação entre a satisfação geral antes e após a facectomia, segundo sexo, idade, escolaridade, estado civil, renda e situação previdenciária dos idosos. Macapá, AP, Brasil, 2013.

\begin{tabular}{|c|c|c|c|c|c|c|c|c|}
\hline \multirow{2}{*}{ Variáveis } & \multirow{2}{*}{$\mathbf{N}$} & \multicolumn{3}{|c|}{ Satisfação geral antes } & \multicolumn{3}{|c|}{ Satisfação geral depois } & \multirow{2}{*}{$\frac{\text { Teste t }}{p}$} \\
\hline & & Média & Med & DP & Média & Med & DP & \\
\hline \multicolumn{9}{|l|}{ Sexo } \\
\hline Masculino & 76 & 58,83 & 59,22 & 15,00 & 65,97 & 66,82 & 12,36 & 0,000 \\
\hline Feminino & 80 & 55,96 & 58,66 & 13,94 & 62,62 & 65,87 & 12,42 & 0,000 \\
\hline \multicolumn{9}{|l|}{ Faixa etária } \\
\hline $60-69$ anos & 71 & 59,74 & 62,15 & 14,20 & 64,65 & 66,11 & 12,65 & 0,001 \\
\hline 70 anos e mais & 85 & 55,37 & 55,28 & 14,52 & 63,92 & 66,18 & 12,38 & 0,000 \\
\hline \multicolumn{9}{|l|}{ Estado Civil } \\
\hline Com companheiro & 26 & 56,01 & 55,82 & 15,00 & 60,62 & 64,31 & 12,93 & 0,067 \\
\hline Sem companheiro & 130 & 57,63 & 59,03 & 14,43 & 64,98 & 66,89 & 12,29 & 0,000 \\
\hline \multicolumn{9}{|l|}{ Analfabeto } \\
\hline Sim & 47 & 51,03 & 49,44 & 13,23 & 59,90 & 59,97 & 11,68 & 0,000 \\
\hline Não & 109 & 60,08 & 60,28 & 14,21 & 66,13 & 68,89 & 12,38 & 0,000 \\
\hline \multicolumn{9}{|l|}{ Renda } \\
\hline Até 1 salário & 148 & 56,94 & 58,06 & 14,34 & 63,77 & 66,11 & 12,44 & 0,000 \\
\hline Mais de 1 salário & 8 & 65,02 & 64,31 & 16,08 & 73,20 & 77,31 & 9,84 & 0,263 \\
\hline \multicolumn{9}{|l|}{ Trabalha } \\
\hline Sim & 16 & 68,76 & 71,81 & 16,03 & 70,88 & 74,10 & 12,40 & 0,609 \\
\hline Não & 140 & 56,02 & 56,44 & 13,81 & 63,48 & 66,11 & 12,32 & 0,000 \\
\hline
\end{tabular}

Verificou-se que independente da idade, escolaridade e sexo, o aumento da satisfação geral foi estatisticamente significante após a cirurgia. Os pacientes sem companheiro (viúvo, divorciado, solteiro), com renda de até um salário mínimo e os aposentados apresentaram melhora significativa da satisfação geral após a cirurgia de catarata, quando comparados aos seus pares.

A Tabela 3 aponta os valores do coeficiente de correlação de Spearman entre os escores da acuidade visual e dos subdomínios do questionário da qualidade de vida. 
Tabela 3: Correlação de Spearman entre os domínios do VQF - 25 e a acuidade visual, antes e depois da facectomia. Macapá, AP, Brasil, 2013.

\begin{tabular}{ccccc}
\hline \multirow{2}{*}{ Subdomínios } & \multicolumn{2}{c}{ Acuidade visual antes (1,23) } & \multicolumn{2}{c}{ Acuidade visual depois (0,57) } \\
\cline { 2 - 5 } & Correlação de Spearman(r) & $\mathbf{P}$ & Correlação de Spearman(r) & $\mathbf{p}$ \\
\hline Saúde geral & $-0,115$ & 0,154 & $-0,096$ & 0,236 \\
Visão & $-0,061$ & 0,451 & $-0,142$ & 0,076 \\
Dor ocular & $-0,037$ & 0,650 & $-0,177$ & 0,027 \\
Atividade perto & $-0,217$ & 0,007 & $-0,144$ & 0,073 \\
Atividade longe & $-0,263$ & $-0,261$ & 0,001 \\
Aspecto social & $-0,099$ & $-0,129$ & 0,108 \\
Saúde mental & $-0,186$ & 0,001 & $-0,199$ & 0,013 \\
Atividades diárias & $-0,202$ & $-0,108$ & 0,181 \\
Dependência & $-0,258$ & 0,021 & $-0,162$ & 0,043 \\
Capacidade de dirigir & $-0,352$ & 0,012 & $-0,075$ & 0,354 \\
Visão de cores & $-0,220$ & 0,001 & $-0,149$ & 0,064 \\
Visão periférica & $-0,262$ & 0,002 & $-0,112$ & 0,163 \\
Satisfação geral & $-0,296$ & 0,006 & $-0,194$ & 0,015 \\
\hline
\end{tabular}

A análise de correlação posterior à facectomia apontou que quanto melhor a acuidade visual do paciente após a cirurgia, maior foi a satisfação em relação à melhora da dor ocular, atividade para longe, saúde mental, dependência e satisfação geral, uma vez que foram constatadas correlações significativas $(p<0,05)$. Porém, destaca-se que todas as associações foram consideradas fracas. Ressalta-se que, comparando as associações antes e depois da facectomia, o único coeficiente que obteve aumento da força de associação foi em relação à dor ocular. A fragilidade na força de associação pode estar relacionada ao curto período de tempo entre a intervenção cirúrgica e a avaliação.

\section{DISCUSSÃO}

As características dos idosos encontradas neste estudo com a maioria na faixa etária de 70 anos ou mais, sexo feminino, analfabetos ou ensino fundamental incompleto, aposentados e renda familiar inferior a um salário mínimo, são compatíveis com outros recentes estudos realizados com idosos ${ }^{(6,15-16)}$, o que confirma o atual perfil dos idosos brasileiros portadores de doenças oculares.

A facectomia refletiu como um fator positivo para a melhora da acuidade visual, a qual passou de 1,23 para 0,57 unidades LogMAR após a cirurgia. Esses resultados equivalem-se aos achados na literatura ${ }^{(5,7,17-18)}$, e constata-se a importância da realização da cirurgia como forma de reabilitação da visão entre os idosos.

A comparação da qualidade de vida antes e após a facectomia revelou uma diferença estatisticamente significativa após o procedimento cirúrgico com médias mais altas encontradas nos domínios "visão geral", "dependência" e "saúde geral".

A saúde geral está diretamente relacionada à saúde ocular, de modo que a recuperação visual é condição determinante para a melhora do bem-estar e aumento da autoestima, mediante o favorecimento de independência e autonomia, participação social, preservação de capacidades cognitivas, hábitos saudáveis e/ou redução de ansiedade e depressão ${ }^{(2,6)}$.

Nessa perspectiva, inclui-se que a cirurgia de catarata proporciona melhora da produtividade no 
trabalho e estimula os indivíduos inativos a procurarem trabalho remunerado, mesmo aqueles com uma idade média superior a $68 \operatorname{anos}^{(19)}$.

Afirma-se que a ausência de saúde ocular tem sido importante influência na ocorrência de prejuízos funcionais. Estudos têm apontado a relação da saúde ocular com a ocorrência de queda, sendo a catarata um fator predisponente em decorrência da dificuldade do indivíduo em enfrentar os obstáculos encontrados no ambiente. Nessa perspectiva, a qualidade de vida de idosos com catarata e que sofreram quedas tem mostrado forte associação com resultados negativos em relação à saúde mental e à dependência para a realização de diversas atividades ${ }^{(20)}$.

Estudo realizado no sertão do Pernambuco, utilizando o questionário VFQ- 25 em idosos, concluiu que o déficit visual trouxe comprometimentos nas atividades de perto e longe e, consequentemente, as atividades de vida diária foram prejudicadas nesses idosos, acarretando maior dependência e insegurança. Tal dependência está ligada a uma pior saúde mental, assim como o prejuízo da vida social, dos relacionamentos com amigos e familiares ${ }^{(6)}$. Esse círculo vicioso reflete o impacto negativo que o déficit visual causa nos idosos. Nesse sentido, a melhora da acuidade visual e consequentemente, o aumento do nível de independência dos idosos podem ser fatores importantes para o aumento do grau de satisfação com a sua saúde geral.

O resultado de um estudo de satisfação após a cirurgia de catarata apontou que a maioria dos pacientes referiu satisfação com a visão geral, pois, após a cirurgia, foi possível ler jornais ou livros, reconhecer pessoas, enxergar sinais e placas de trânsito, preencher cheques e formulários, praticar esportes, cozinhar, assistir à televisão e se barbear ou se maquiar, demonstrando uma boa qualidade de vida ${ }^{(2)}$.

Outros estudos nacionais e internacionais que utilizaram o questionário VFQ-25 em idosos concluíram que a percepção do ambiente prejudicada pela acuidade visual diminuída tende a levar os idosos a manifestar insegurança para determinadas atividades ${ }^{(15,20)}$. Isso influencia o agravamento do atual estado de saúde dos idosos, que, além das doenças características da senilidade, podem ser acometidos por eventuais acidentes, durante a realização de suas atividades habituais, comprometendo ainda mais a saúde e qualidade de vida.

Conhecer a percepção dos pacientes submetidos à facectomia sobre os domínios da qualidade de vida pode fornecer indicadores para a equipe de saúde e direcionar as orientações específicas para cada paciente. Neste estudo, o aspecto social foi à dimensão melhor pontuada pelos idosos, surgindo como aspecto favorável que pode ser utilizado pela equipe de saúde para apoiar ações de intervenção que auxiliem na melhoria das dimensões de saúde mental e atividades diárias, identificadas com baixos escores.

$\mathrm{O}$ isolamento, a confiança e as conexões sociais são fatores considerados preditores de resultados de saúde mental, independente da faixa etária do indivíduo, sendo o isolamento social o fator que gera mais impacto na saúde mental do idoso(21). Desse modo, identificar fragilidades e fortalezas nas dimensões relacionadas à qualidade de vida dos idosos pode subsidiar a equipe de saúde na adotação de ações mais integrativas e individualizadas, atendendo às reais necessidades de cada paciente.

Nesse contexto, a enfermagem ganha destaque relevante, apesar de não ser uma área contida na 
grade curricular dos cursos de graduação, o que implica na necessidade de avançar na construção de significados e responsabilidades do enfermeiro em oftalmologia, a fim de contribuir com a resolutividade e segurança da assistência oftalmológica.

Uma ferramenta que pode ser utilizada para o acompanhamento de pacientes no pós-operatório da facectomia e que independe do espaço geográfico é o telemonitoramento. A ação consiste em acompanhamento do paciente por telefone e seu uso tem demonstrado melhora na adesão ao tratamento, continuidade dos cuidados pós-operatórios no domicílio, além de permitir redução da ansiedade entre os pacientes $^{(22)}$. Estratégias como essas devem ser instituídas no contexto da prática profissional a fim de garantir a avaliação das intervenções de saúde.

Este estudo e muitos outros estudos desenvolvidos no país ${ }^{(23)}$ e no mundo ${ }^{(5)}$ tornam evidente o grande número de pacientes com déficit visual devido à catarata, com baixo grau de instrução e baixa renda familiar, enfatizando a necessidade de programas na saúde pública que facilitem o acesso à informação e ao tratamento da doença. Avaliar a percepção dos idosos em relação à qualidade de vida antes e após a intervenção cirúrgica permitiu o levantamento de indicadores assistenciais que direcionam a tomada de decisão no planejamento terapêutico em oftalmologia.

Salienta-se que a recuperação da visão por cirurgia produz benefícios econômicos e sociais ao indivíduo, à sua família e à comunidade. No contexto de avaliação dos serviços de saúde, o presente estudo possibilitou breve análise sobre a importância de parcerias entre centros de referência e sistemas de saúde interestaduais para a melhoria de problemas de saúde pontuais dos indivíduos. Porém, a situação precária de acesso à assistência oftalmológica, devido aos obstáculos encontrados pelo próprio sistema de saúde, dificulta a utilização do recurso cirúrgico e da assistência especializada. Entre as dificuldades encontradas podem ser citados o uso de equipamentos e técnicas desatualizadas e a falta de profissionais médicos especializados em algumas regiões ${ }^{(9)}$. Na região do Amapá, contexto em que se desenvolveu este estudo, destaca-se o número reduzido de profissionais e serviços especializados em oftalmologia, que se beneficiam de campanhas comunitárias para o acesso ao atendimento e tratamento oftalmológicos.

Estudos apontaram a fila de espera da cirurgia no Sistema Único de Saúde, o custo da lente intraocular no sistema privado e o difícil acesso geográfico ao tratamento ${ }^{(5,19-21)}$ como as principais barreiras para a não realização de cirurgia de catarata. Diante da impossibilidade de reabilitação da visão, como consequência, muitos idosos abandonam seus empregos tornando-se indivíduos economicamente inativos.

Mais do que fazer uma intervenção, é necessário acompanhar o seu efeito na vida das pessoas. 0 desafio ora proposto é a imersão dos profissionais de enfermagem no acompanhamento e avaliação dos pacientes oftalmológicos, com ações terapêuticas mais incisivas e resolutivas, com a finalidade de restabelecer a qualidade de vida do idoso.

\section{CONCLUSÃO}

A partir do estudo foi possível verificar a influência da saúde ocular e a qualidade de vida dos idosos. 
Constatou-se diferença estatisticamente significativa na percepção do idoso, revelando que a facectomia está associada a um impacto positivo sobre acuidade visual e, consequentemente, na qualidade de vida.

Como limitação, pode-se dizer que foi feita avaliação pós-operatória no 15 dia com melhora da visão, mas não foi feita avaliação de acuidade visual corrigida, o que poderia apontar melhor satisfação do paciente. Sugere-se, para próximos estudos, que a acuidade visual seja realizada após o exame de refração, ou seja, acuidade visual corrigida, após 180 dias do procedimento cirúrgico.

De todo modo, o presente estudo trouxe importantes indicadores relacionados à saúde ocular e à qualidade de vida, evidenciando aspectos de impacto na percepção da saúde geral do idoso após a facectomia. Entretanto, também apontou áreas que exigem intervenção multiprofissional para promover o restabelecimento da saúde mental e melhorar o desenvolvimento de atividades diárias. Nessa direção, sugerem-se, ainda, estudos que avaliem o impacto da saúde ocular na progressão de problemas funcionais em idosos.

A campanha assistencial de catarata se mostrou útil para diminuir a inacessibilidade ao tratamento oftalmológico na saúde pública e favoreceu a recuperação da visão dos idosos. Os dados deste estudo poderão servir para orientação de novas políticas públicas de promoção da saúde dos portadores de catarata e, consequentemente, contribuir para a melhoria da saúde e da qualidade de vida dos idosos.

\section{REFERÊNCIAS}

1. Malagutti W. Cuidados de Enfermagem em Geriatria. 1a ed. Rio de Janeiro: Editora Rubio; 2013.

2. Santos BWL, Cançado JEP, Ferraz VAS, Campos M. Avaliação da qualidade de vida em pacientes submetidos à cirurgia de catarata, com implantes de lentes monofocais bifocais e multifocais. Rev Bras Oftalmol [Internet]. 2014 [acesso em: 22 ago. 2017];73(2):86-92. Disponível em: http://dx.doi.org/10.5935/0034-7280.20140020.

3. Conselho Brasileiro de Oftalmologia. Catarata: Diagnóstico e tratamento. Projeto Diretrizes [Internet]. São Paulo: Associação Médica Brasileira/Conselho Federal de Medicina; 2003 [acesso em: 22 ago. 2017]. Disponível em:

https://diretrizes.amb.org.br/_BibliotecaAntiga/catarata-diagnostico-e-tratamento.pdf.

4. Chi MJ, Lee CY, Wu SC. The prevalence of chronic conditions and medical expenditures of the elderly by chronic condition indicator (CCI). Arch Gerontol Geriatr [Internet]. 2011 [acesso em: 22 ago. 2017];52(3):284-9. Disponível em: https://doi.org/10.1016/j.archger.2010.04.017.

5. Alvarado Ds, Rivera B, Lagos L, Ochoa M, Starkman I, Castillo M et al. Encuesta nacional de ceguera y deficiencia visual evitables en Honduras. Rev Panam Salud Publica [Internet]. 2014 [acesso em: 22 ago. 2017];36(5):300-5. Disponível em: http://www.scielosp.org/scielo.php?script=sci_arttext\&pid=S1020-49892014001000003\&Ing=es. 6. Bravo Filho VTF, Ventura RU, Brandt CT, Sarteschi C, Ventura MC. Impacto do déficit visual na qualidade de vida em idosos usuários do sistema único de saúde vivendo no sertão de Pernambuco. Arq Bras Oftalmol [Internet]. 2012 [acesso em: 22 ago. 2017];75(3):161-5. Disponível em: http://dx.doi.org/10.1590/S0004-27492012000300002. 7. Pereira MCSR, Krieger MAL, Mariushi AC, Moreira H. Perfil epidemiológico de pacientes com catarata traumática no Hospital de Olhos do Paraná. Rev Bras Oftalmol [Internet]. 2012 [acesso em: 22 ago. 2017];71(4):236-40. Disponível em: http://dx.doi.org/10.1590/S0034-72802012000400006.

8. Campolina AG, Dini PS, Ciconelli RM. Impacto da doença crônica na qualidade de vida de idosos da comunidade em São Paulo (SP, Brasil). Cien Saude Colet [Internet]. 2011 [acesso em: 22 ago. 2017];16(6):2919-25. Disponível em: http://dx.doi.org/10.1590/S1413-81232011000600029. 
9. Kara-Junior N, Santhiago mr, Espindola RF. Facoemulsificação versus extração extracapsular no sistema público de saúde: análise de custos para o hospital, para o governo e para a sociedade. Rev Bras Oftalmol [Internet]. 2012 [acesso em: 22 ago. 2017];71(2):115-24. Disponível em: http://dx.doi.org/10.1590/S0034-72802012000200009. 10. Ciosak SI, Braz E, Costa MFBNA, Nakano NGR, Rodrigues J, Alencar RA et al. Senescência e senilidade: novo paradigma na atenção básica de saúde. Rev Esc Enferm USP [Internet]. 2011 [acesso em: 22 ago. 2017];45(spe2):17638. Disponível em: http://dx.doi.org/10.1590/S0080-62342011000800022.

11. Mangione CM, Lee PP, Gutierrez PR, Spritzer K, Berry S, Hays RD. Development of the 25-list-item National Eye Institute Visual Function Questionnaire. Arch Ophthalmol [Internet]. 2001 [acesso em: 22 ago. 2017];119(7):1050-8. Disponível em: http://dx.doi.org/10.1001/archopht.119.7.1050.

12. Ferraz EVAP, Lima CA, Cella W, Arieta CEL. Adaptação de questionário de avaliação da qualidade de vida para aplicação em portadores de catarata. Arq Bras Oftalmol [Internet]. 2002 [acesso em: 22 ago. 2017];65(3):293-8. Disponível em: http://dx.doi.org/10.1590/S0004-27492002000300002.

13. Simão LM, Lana-Peixoto MA, Araújo CR, Moreira MA, Teixeira AL. The Brazilian version of the 25-Item National Eye Institute Visual Function Questionnaire: translation, reliability and validity. Arq Bras Oftalmol [Internet]. 2008 [acesso em: 22 ago. 2017];71(4):540-6. Disponível em: http://dx.doi.org/10.1590/S0004-27492008000400014.

14. Guilford JP. Fundamental statistics in psychology and education. 4a ed. New York: McGraw-Hill; 1950. 605 p. 15. Macedo BG, Pereira LSM, Rocha FL, Castro ANBV. Brandão Vilela de. Medo de cair e qualidade de vida em idosos com catarata. Rev. bras. geriatr. gerontol. [Internet]. 2013 [acesso em: 22 ago. 2017];16(3):569-77. Disponível em: http://dx.doi.org/10.1590/S1809-98232013000300014.

16. Jannuzzi FF, Cintra FA, Rodrigues RCM, São-João TM, Gallani MCBJ. Medication adherence and quality of life among the elderly with diabetic retinopathy. Disponível em [Internet]. 2014 [acesso em: 22 ago. 2017];22(6):902-10. Disponível em: http://dx.doi.org/10.1590/0104-1169.3477.2494.

17. Corrêa EP, Oliveira LFL, Serracarbassa PD, Oshima A, Castro EFS. Avaliação do edema macular após cirurgia não complicada de facoemulsificação com implante de lente intraocular por meio da tomografia de coerência óptica spectral domain. Arq Bras Oftalmol [Internet]. 2013 [acesso em: 22 ago. 2017];76(6):357-62. Disponível em: http://dx.doi.org/10.1590/S0004-27492013000600008.

18. Násser LS, Santos Neto PE, Paranaíba LMR, Ribeiro LMG, Figueiredo LP, Martelli Júnior H. Cirurgia combinada de catarata e glaucoma com ponto escleral perilímbico: técnica cirúrgica e resultados a longo prazo. Rev Bras Oftalmol [Internet]. 2012 [acesso em: 22 ago. 2017];71(4):241-4. Disponível em: http://dx.doi.org/10.1590/S003472802012000400007.

19. Kara-Junior N, Parede TRR, Santhiago MR, Espindola RF, Mazurek MGG, Carvalho RS. Custo social de duas técnicas de cirurgia de catarata no Brasil. Rev Saude Publica [Internet]. 2010 [acesso em: 22 ago. 2017];44(5):957-62.

Disponível em: http://dx.doi.org/10.1590/S0034-89102010005000025.

20. Yamada M, Mizuno Y, Miyake Y. A multicenter study on the health-related quality of life of cataract patients: baseline data. Jpn J Ophthalmol [Internet]. 2009 [acesso em: 22 ago. 2017];53(5):470-6. Disponível em: http://dx.doi.org/10.1007/s10384-009-0709-0.

21. Kara-Júnior N, Dellapi Jr R, Espíndola RF. Dificuldades de acesso ao tratamento de pacientes com indicação de cirurgia de catarata nos Sistemas de Saúde Público e Privado. Arq Bras Oftalmol [Internet]. 2011 [acesso em: 22 ago. 2017];74(5):323-5. Disponível em: http://dx.doi.org/10.1590/S0004-27492011000500002

22. Delphino TM, Souza PA, Santana RF. Telemonitoramento como intervenção no pós-operatório de facectomia: revisão sistemática da literatura. REME [Internet]. 2016 [acesso em: 22 ago. 2017];20:e937. Disponível em: http://dx.doi.org/10.5935/1415-2762.20160007.

23. Taicher PH, Thorell MR, Santana DC, Garciolli G, Nunes CM, Marcon IM. Perfil demográfico dos pacientes atendidos na Campanha de Catarata da Santa Casa de Porto Alegre. Rev Bras Oftalmol [Internet]. 2010 [acesso em: 22 ago. 2017];69(2):89-93. Disponível em: http://dx.doi.org/10.1590/S0034-72802010000200004. 